\title{
Aprendizaje Basado en Proyectos en el marco STEM. Componentes didácticas para la Competencia Científica
}

\author{
Jordi Domènech-Casal ${ }^{1,2}$ \\ ${ }^{1}$ Institut Marta Estrada (Granollers), ${ }^{2}$ Departamento de Didáctica de las Matemáticas y \\ las Ciencias Experimentales, Universitat Autònoma de Barcelona.
}

[Recibido el 3 de octubre de 2018, aceptado el 24 de octubre de 2018]

El Aprendizaje Basado en Proyectos (ABP) es un enfoque metodológico que promueve el aprendizaje de los conceptos científicos mediante su instrumentalización en la resolución de un problema o elaboración de un producto. El diseño de actividades ABP implica complejidades en la relación entre contextos y contenidos, y el grado de desarrollo del discurso propio de las áreas científicas en relación a la interdisciplinariedad y apertura del proyecto. Se propone una herramienta de análisis de componentes didácticas para proyectos ABP STEM en relación al impacto en la Competencia Científica.

Palabras clave: Aprendizaje Basado en Proyectos; STEM; Modelo; Contexto

\section{Project-Based Learning for STEM. Didactic components for Scientific Competence}

Project-Based Learning is a methodology promoting learning of scientific concepts through its instrumentalization in solving a problem or constructing a product. Designing PBL activities entails complex relationships between contexts, contents and the development of science discourse regarding interdisciplinarity and openess of projects. We propose a tool for the analysis of didactic components of STEM PBL activities for its impact on scientific competence and discuss its applications.

Keywords: Project-Based Learning; STEM; Model; Context

Para citar el artículo. Domènech-Casal, J. (2018). Aprendizaje Basado en Proyectos en el marco STEM. Componentes didácticas para la Competencia Científica. Ápice. Revista de Educación Científica, 2(2), 29-42. DOI: https://doi.org/10.17979/arec.2018.2.2.4524

Contacto.jdomen44@xtec.cat 


\section{Introducción}

\section{Componentes didácticas en el despliegue de la Competencia Científica}

Definir Competencia Científica es algo complejo. La definición propuesta por la OCDE, que sintetiza distintas propuestas anteriores, es bastante operativa, al menos en lo relativo al diseño de actividades de aula. La OCDE (2013) propone que la Competencia científica estaría formada por tres dimensiones, conceptual procedimental y epistémica. Las describimos a continuación asociadas a componentes didácticas para la enseñanza de las ciencias.

Como dimensión conceptual entenderíamos la capacidad utilizar modelos científicos para la interpretación de fenómenos naturales o contextos, o relacionar distintos modelos entre sí. Es difícil definir "modelo" o "contexto" y en la práctica en el aula a menudo suelen identificarse con los "conceptos" y las "situaciones" en que se ubican las actividades de aprendizaje. Varios autores proponen que el aprendizaje de los conceptos científicos debe producirse a partir de contextos auténticos (Gilbert, 2006, Sanmartí, Burgoa y Nuño, 2011, Blanco, España y Rodríguez, 2012) para resultar en un dominio de los conceptos científicos suficiente para su transferencia a otros contextos (Gilbert, Bulte y Pilot, 2011). Definir con precisión qué podemos considerar contexto es complejo, pero algunos autores (Duranti y Goodwin, 1992, Gilbert, 2006) entienden que debe incluir un escenario social, un ambiente o marco conductual, un lenguaje específico y una relación con el conocimiento extrasituacional. Existe un consenso que en el trabajo en contexto es una vía privilegiada para la competencia científica, si bien el encaje de modelos y contextos en actividades didácticas no es evidente (Blanco, España y Rodríguez, 2012, Kortland, 2007, Couso, 2015).

La dimensión procedimental refiere a la capacidad de usar habilidades de razonamiento científico, como identificar pautas, inducir, deducir, o diseñar experimentos. Algunos autores identifican la dimensión procedimental con las "Inquiry skills", si bien la oportunidad de ese término en relación a la competencia científica es discutido (Couso, 2014). En su traducción a las actividades de aula, eso implica que debe existir algún conflicto que requiera el uso de estas habilidades de razonamiento. Podemos definir dos tipos de conflictos: conflictos entre lo que sabemos y lo que se desprende de los fenómenos (solemos llamarlos conflictos cognitivos) y conflictos entre lo que tenemos y lo que queremos hacer. El primero es muy propio de las actividades de Indagación y Modelización, en las que el conflicto cognitivo actúa de motor para avanzar en la elaboración de Leyes que describan cómo se comporta un sistema (Indagación) o de Modelos que expliquen por qué lo hace así (Modelización) (Bybee, 1997, Caamaño, 2011, Windschilt et al, 2008, Couso, 2015).

El segundo tipo es muy propio del Aprendizaje Basado en Proyectos, en los que un conflicto externo al aprendizaje (construir un barco, diseñar un parque) actúa como contexto para el desarrollo instrumental de modelos científicos y habilidades de razonamiento (Sanmartí y Márquez, 2017, Domènech-Casal et al, 2018a).

La dimensión epistémica se asocia al modo que tiene la ciencia de validar el conocimiento. Por ello, implica una cierta apropiación del Discurso de la ciencia, y suele reclamar el desarrollo de capacidades de uso de pruebas y argumentación (Bravo, Puig y Jiménez-Aleixandre, 2009), pero también de ideas sobre la Naturaleza de la Ciencia como conocimiento parcial, consensual basado en pruebas, provisional y resultado de un proceso histórico. Distintos enfoques, como las Controversias científicas o socio-científicas (Kolst $\varnothing, 2001$, Sadler, 2009, Díaz y Jiménez-Liso, 2012), la Historia de la Ciencia (AcevedoDíaz y García Carmona, 2017) o el trabajo sobre Pseudociencias (Vieira, Tenreiro-Vieira y Martins, 2010, García-Molina, 2015), ofrecen contextos o situaciones de aula interesantes para ayudar al alumnado a entender la necesidad de saber usar perspectivas epistémicas. 
En el diseño de actividades didácticas, el papel que juegan distintas componentes (los contenidos, contextos, conflicto y discurso de la ciencia) es clave para las aportaciones al despliegue de la competencia científica y complejo de orquestar.

\section{La irrupción STEM}

El informe Rocard (Rocard et al, 2006) advertía de algo que se ha convertido en una preocupación global en los países desarrollados: no hay suficientes vocaciones científico-tecnológicas entre el alumnado, y el problema se acrecienta en relación al género y el origen socio-económico: las alumnas y el alumnado de origen socio-económico humilde accede en muy baja proporción a las vocaciones científico-tecnológicas. Este es un fenómeno que puede tener en un futuro un gran impacto económico (por dificultades en el desarrollo de políticas de innovación y transferencia tecnológica) y está teniendo ya en el presente efectos muy negativos en la equidad y en la amplitud del espectro tecnológico (p.e.: tener pocas mujeres ingenieras es un sesgo de género que reduce enormemente la capacidad de la ingeniería de ofrecer respuestas distintas y amplias a problemas nuevos). Se ha detectado la necesidad de un empuje desde el espacio educativo, y desde entonces, el término STEM (acrónimo de la expresión en inglés "Science, Technology, Engineering and Mathematics") ha venido siendo cada vez más presente en el mundo profesional de la enseñanza de las ciencias (formación de profesorado, programas de innovación), gracias al impulso recibido por distintos proyectos gubernamentales financiados a nivel nacional o internacional, con un doble objetivo: las vocaciones científico-tecnológicas y una educación para una ciudadanía competente científica y tecnológicamente.

En su entrada en el espacio educativo, se ha hecho un esfuerzo por dotar de significado didáctico y pedagógico el término STEM (Bybee, 2010, Brown et al, 2011, Couso, 2017). En la mayoría de casos se ha optado por identificar STEM con la interdisciplinariedad, obviando que la pedagogía ya tenía un término para eso ("interdisciplinar", o "trabajo integrado"). La incorporación de la A (por Artes) en ese acrónimo como "STEAM" ha hecho -si cabe- el panorama más complejo. A veces, " $A$ " significa Artes en significado estricto (música, danza, pintura, música,...), a veces en significado amplio (desde la literatura a la filosofía, lo que conocemos como Humanidades). Además, la inclusión de la "A" se ha reclamado en ocasiones como modo de incorporar creatividad en las propuestas STEM. Eso ha generado no pocas susceptibilidades, al sugerir que las demás áreas no tienen sus propios espacios de creatividad, produciéndose fratricidas batallas entre defensores de STEM y de STEAM. Aun así, esa definición continúa cuadrando mal con la incorporación de la robótica y la programación como estandartes STEM sin ser interdisciplinares, y distintas opciones educativas nada recientes (uso de las TIC, del ABP o de utillaje de laboratorio) se han asociado también con ese acrónimo. En su concreción en la práctica, el término STEM parece actuar como un paraguas poco selectivo en el que también se cobijan las Ilamadas "soft skills" o habilidades prácticas vinculadas a la autonomía, como el trabajo en equipo, la creatividad o el pensamiento computacional (Couso, 2017).

Desde nuestro punto de vista, la causa de que sea tan difícil dotar de significado pedagógico el término STEM es que en realidad no lo tiene: STEM es un término que representa un objetivo político, no un enfoque pedagógico o didáctico, aunque pueda promoverse desde enfoques pedagógicos o didácticos. En este sentido, lo honesto y práctico es considerar STEM todos aquellos enfoques (precedentes o de nueva creación) que puedan ser útiles para la consecución de los objetivos STEM (Vocaciones, sesgos de género y origen socioeconómico, ciencias para la ciudadanía). Ello incluiría no sólo metodologías (como el ABP y la indagación), sino también tecnologías (como la robótica y las Apps) y muy especialmente perspectivas (como la ciudadanía, la perspectiva de género y la sostenibilidad) (Acevedo-Díaz, 2004, Vilches, Gil y Cañal, 2010, Couso, 2017, Domènech-Casal, 2018a). 
Esto libera a la didáctica y la pedagogía de un encargo sobrevenido (intentar crear una narración que justifique pedagógicamente ese término político) y permite que aporte aquello que puede aportar: el conocimiento sobre cómo se aprende, los métodos y sus limitaciones. ¿Pretende STEM una ciudadanía más capaz de tomar decisiones en contextos participados por la ciencia y la tecnología? Veamos cómo. ¿Conecta STEM con la necesidad de generar actitudes hacia la ciencia y la tecnología? Veamos cómo.

En este sentido, es innegable que los objetivos STEM requieren el desarrollo de la Competencia científica y la Competencia tecnológica, algo en que, efectivamente, desde la didáctica se pueden hacer aportaciones. En todo caso, la emergencia de espacios y proyectos STEM genera la necesidad de evaluar qué aportaciones a las distintas dimensiones de la Competencia científica realizan esas actividades y de qué modo pueden convertirse en vías para su despliegue.

\section{El Aprendizaje Basado en Proyectos como método candidato}

De forma concomitante con la irrupción STEM, el Aprendizaje Basado en Proyectos (ABP) está en los últimos años generando expectativas como "forma" en la que encarnar en las aulas el discurso teórico de la enseñanza por competencias (Sanmartí, 2016). El Aprendizaje Basado en Proyectos es una metodología didáctica nada reciente, propuesta como tal por Kilpatrick (1918) hace ya un siglo. En la actualidad, el término ABP ha ido cobrando distintos significados, que acostumbran a incluir (Domènech-Casal et al, 2018a):

- La enseñanza se produce en el contexto de una situación o problema.

- Los contenidos clave se aprenden a partir del contexto y la actuación.

- Se parte de un reto o propósito compartido por el alumnado.

Kilpatrick (1918) establece que ese propósito compartido por el alumnado permite distinguir entre 4 categorías de proyectos: Elaborar un producto, Resolver un problema, Disfrutar de una experiencia estética y Obtener un conocimiento. Consideramos que estas 4 categorías pueden agruparse en dos tipos principales según la instancia que impulsen (Tabla 1).

Tabla 1. Instancias y ejemplos de proyectos según las categorías de Kilpatrick, según la instancia

\begin{tabular}{|l|l|l|}
\hline Instancia & Tipos de Kilpatrick & Ejemplos de Metodologías \\
\hline Queremos hacer... & Elaborar un producto & "Design Thinking" \\
\cline { 2 - 3 } & Resolver un problema & $\begin{array}{l}\text { Controversias } \\
\text { Estudio de Casos } \\
\text { Aprendizaje-Servicio } \\
\text { Ciencia Ciudadana }\end{array}$ \\
\cline { 2 - 3 } & $\begin{array}{l}\text { Disfrutar de una experiencia } \\
\text { estética }\end{array}$ & STE[A]M \\
\hline Queremos saber... & Obtener un conocimiento & Indagación y Modelización \\
\hline
\end{tabular}

Las tres primeras categorías se identifican con la instancia "Queremos hacer" (DomènechCasal et al, 2018a). En ellas, existe un objetivo externo al conocimiento (construir un barco, ordenar el tráfico de una ciudad, disfrutar de una observación astronómica) que actúa como propósito para la construcción instrumental de conceptos o modelos y el desarrollo de aspectos procedimentales y epistémicos. Las reformulaciones actuales del ABP se corresponden con esa instancia y, en particular, a las categorías de Elaborar un Producto o Resolver un Problema (Larmer, Mergendoller y Boss, 2015). Esas dos categorías de proyectos se corresponden con enfoques ya conocidos o de nuevo cuño, como el Aprendizaje- 
Servicio (Martín, 2016) o el "Design Thinking" (Albalat, 2017). Igualmente, dentro de la categoría Resolver un problema podríamos ubicar el llamado "Aprendizaje Basado en Problemas", que en la enseñanza de las ciencias adopta formas como las Controversias Socio-Científicas (Sadler, 2009), la Ciencia Ciudadana o los Estudios de Caso (DomènechCasal, 2017a).

La última categoría reúne los proyectos con la instancia "Queremos saber" en los que el propósito coincide con el objetivo de aprendizaje ("Queremos saber cómo se alimentan las plantas") y en la enseñanza de las ciencias encuentra exponentes en la Indagación y la Modelización (Windschitl, Thompson y Braaten, 2008).

El ABP ha sido valorado como una metodología privilegiada para el despliegue de los objetivos STEM, en forma de actividades de Aprendizaje Basado en Proyectos en las que participan dos o más materias del ámbito STEM. Esto hace que -añadida a la complejidad de la definición de STEM- se incorporen en esta fórmula las complejidades propias de la metodología $\mathrm{ABP}$, configurándose distintas componentes a tener en cuenta en relación al diseño de proyectos ABP STEM.

Por un lado aspectos relacionados con componentes de la Competencia científica que hemos citado en la introducción: Si bien es complejo definir las relaciones entre lo que Kilpatrick propone como "propósito" y lo que Gilbert propone como "contexto", es inevitable asociar en el ABP de alguna forma lo uno con lo otro. En los proyectos, los conceptos o modelos no siempre están perfectamente encajados, entre sí y con los conflictos a resolver. En ocasiones los propósitos y contextos requieren sólo un nivel de conocimiento muy superficial de los conceptos, por lo que se aprenden de forma parcial o poco sofisticada, o el conflicto a resolver no instrumentaliza el uso de conceptos ni la comprensión o análisis de los contextos. Además, en ocasiones por un diseño incorrecto, en ocasiones debido a contextos excesivamente complejos, puede resultar difícil desplegar el discurso de la ciencia (Domènech-Casal et al, 2018a).

Añadido a esto, el ABP suele implicar la participación del alumnado en la planificación (resultando proyectos con mayor o menor grado de apertura) y evaluación del proyecto (Sanmartí y Márquez, 2017, Grau, 2009). Si bien esto parece bastante razonable para el desarrollo de las "soft skills" y la implicación del alumnado (algo muy en consonancia con los objetivos STEM), supone un reto en lo referente al papel de contenidos, conflicto, contexto y discurso de la ciencia en el diseño de propuestas didácticas (Miró et al, 2016, Simarro y Couso, 2016, Sanmartí y Márquez, 2017). La participación del alumnado en la toma de decisiones puede "desplazar" partes o todo este conjunto (contenido, contexto, conflicto) de los espacios didácticos en que su uso redunda en un mejor aprendizaje.

Dada la urgencia con la que se están implantando las iniciativas STEM y la metodología $A B P$, son necesarias herramientas que ayuden a analizar esas propuestas ABP-STEM desde el punto de vista de la Competencia científica. Estas herramientas deberían considerar las componentes didácticas vinculadas a la Competencia Científica y el papel de la interdisciplinariedad y apertura de las actividades, con vistas a identificar qué características cumplen las actividades que promueven la Competencia científica. En este trabajo nos hemos propuesto como objetivos elaborar una herramienta de evaluación de proyectos ABP STEM y testarla en proyectos aplicados en las aulas.

\section{Metodología}

Como punto de partida, hemos definido componentes didácticas relevantes en el diseño de proyectos ABP STEM. Estas componentes se han definido a partir de los retos y complejidades propios del ABP descritos en la introducción (Contexto, Conflicto, Discurso, 
Contenido, Apertura). En esta definición, aunque "Contenido" es un término con un significado muy amplio, se ha elegido para designar "Conceptos" o "Modelos" científicos. Como sexta componente, se ha considerado importante incluir también la Interdisciplinariedad. Esta última componente se ha incluido porque, sin ser imprescindible en el ABP, sí tiene implicaciones importantes en el diseño de propuestas didácticas y -como se ha descrito en la introducción- es una referencia usual en la definición de STEM. Hemos elaborado una rúbrica (que hemos llamado ABPMap) en la que hemos creado una hilera para cada componente y establecido tentativamente 4 niveles de despliegue de cada componente (Tabla 2). Esos niveles de despliegue se han creado partiendo de descripciones de proyectos en el marco STEM-ABP disponibles en la bibliografía (Domènech-Casal, 2017b, 2017c). Se han analizado las descripciones de los proyectos, identificado su despliegue máximo y mínimo para cada componente y graduado los desarrollos intermedios en dos niveles más, obteniendo para cada componente 4 niveles de despliegue. Por ejemplo, en relación a la componente "Apertura" se han encontrado propuestas ABP en las que el alumnado decide temática y planificación del proyecto (lo que hemos considerado el nivel máximo de despliegue de esa componente) y otras en las que el alumnado no decide absolutamente nada y consisten en una sucesión de tareas cerradas (lo que hemos considerado el nivel mínimo de despliegue). Entre un tipo y otro, hemos encontrado distintos proyectos en los que el alumnado participa en distintos grados en la toma de decisiones en distintos aspectos (evaluación, planificación), que hemos diferenciado en dos niveles intermedios. Se ha procedido de modo equivalente para las demás componentes hasta completar la propuesta de rúbrica.

Una vez elaborada la rúbrica, la hemos usado para analizar ocho proyectos de ámbito STEM elegidos por haberse implementado en las aulas, encontrarse disponible una descripción a fondo de su diseño y aplicación y por corresponder a distintas tipologías de la taxonomía ABP propuesta en la introducción. Se ha valorado para cada proyecto su aportación a cada una de las componentes didácticas definidas en la rúbrica, ubicando con un punto su posición en cada hilera de la rúbrica. Esto ha permitido (uniendo los puntos de cada proyecto) trazar una línea para cada proyecto como representación de su perfil didáctico.

\section{Descripción de los proyectos analizados}

Proyecto Montgolfier tournament (Domènech-Casal, 2018b)

Proyecto orientado a la enseñanza de los cambios y tipos de energía del tipo "Elaborar un producto". El alumnado diseña y construye un globo aerostático para participar en una competición. En el proceso, participa en distintos eventos de indagación y, así como procesos dirigidos a elegir materiales y combustibles en relación a su masa y energía química.

\section{Proyecto CRASH (Domènech et al, 2018b)}

Proyecto del tipo "Resolver un problema" (Estudio de caso) en el que el alumnado debe actuar como perito judicial para determinar, instrumentalizando conceptos de Cinemática y Dinámica, qué narraciones de distintos accidentes son físicamente posibles. A lo largo del proyecto, el alumnado toma distintas decisiones y argumenta en base a pruebas sus decisiones.

Proyecto EXOS (Ruiz, Llorente y Domènech-Casal, 2017)

El alumnado participa en una actividad de Indagación y Modelización en la que parte de distintas pruebas (tránsitos planetarios, espectros) y el uso de distintos instrumentos (simuladores de astronómicos) para determinar las características de distintos Exoplanetas 
y elaborar un artículo periodístico narrando sus descubrimientos. En el proceso, el alumnado aprende sobre el comportamiento de los cuerpos celestes y métodos de estudio de la astronomía.

\section{Proyecto Mission to Stars (Domènech-Casal y Ruiz-España, 2017)}

Se encarga al alumnado la elaboración de un producto, la propuesta técnica y económica de una misión de investigación astronómica. El alumnado formula preguntas investigables, identifica y ubica en la geografía del universo los objetos que deben analizarse para responderlas y diseña el equipamiento y rutinas informáticas de un telescopio orbital para llevar a cabo la misión. El proyecto termina con una presentación destinada a conseguir la financiación para su proyecto de investigación.

Proyecto Retorno a Karlsruhe (Domènech-Casal, 2019)

Se proponen al alumnado distintas tarjetas representando distintos elementos químicos. El alumnado debe idear un modo de ordenarlas, emulando sin saberlo el proceso histórico de la construcción de la Tabla Periódica e instrumentalizando distintos contenidos (estructura del átomo, distribución electrónica, enlace químico,..) que se trabajan en paralelo en forma de Estudio de Caso. La actividad concluye con un congreso científico final en el que el alumnado debe realizar predicciones de elementos químicos que faltan en su propuesta y sus propiedades físico-químicas.

\section{Proyecto GeaTours (Domènech-Casal, 2014)}

El alumnado se organiza en equipos que actúan como responsables de una agencia de viajes geológicos. El producto a elaborar es un tríptico que incluya 8 formaciones geológicas siguiendo alguna línea argumental de su elección. Para ello, deben usar distintas técnicas de representación y estudio geológicas y matemáticas (perfiles, curvas de nivel, uso de coordenadas GPS, tipos de formaciones...) que sean de utilidad para la representación de las formaciones en el Tríptico. El alumnado consensua qué criterios evaluar en el Tríptico, relativos al formato y el contenido.

\section{Proyecto Diseñemos un envase (Planella et al, 2017)}

El alumnado recibe el encargo de organizarse en equipos de innovación de una empresa y diseñar un envase para un alimento. El alumnado diseña la imagen de marca, decide los materiales y busca estrategias para establecer la forma para contener un volumen determinado. Cada alumno debe elaborar junto con el envase un informe sobre el proceso y resultado final y relacionarlo con los aprendizajes y presentar a sus compañeros en formato comercial las características de su envase y su justificación matemática, comercial y tecnológica (que es coeavaluada). Todo el grupo clase calcula y comprueba los cálculos de volumen de cada envase.

\section{Proyecto Se ha escrito un Crimen (Redondo et al, 2017)}

El proyecto se inicia con una noticia en la prensa que informa de la aparición de un cadáver en el instituto. La policía local presenta el caso al alumnado, que a lo largo de distintas tareas cerradas analiza y acumula evidencias sobre el caso, junto con distintos testimonios. El alumnado decide el orden en que realiza las tareas y analiza muestras biológicas, tratamiento de imagen digital, contexto socio-económico, análisis de materiales... y debe plasmar sus conclusiones en formato de foto-novela y grabar un vídeo-reconstrucción del crimen. Los docentes participantes cualifican mediante distintas rúbricas las tareas y productos del alumnado. 


\section{Resultados}

El proceso de análisis de los distintos proyectos ha implicado observar el proyecto desde cada una de las componentes propuestas de forma independiente. Aunque inicialmente algunas componentes parecían estar asociadas entre sí en algunos proyectos (lo que sugería fusionar algunas de ellas), en otros proyectos tenían valores distintos. Por ejemplo, en el proyecto Montgolfier tournament, aunque se propone al alumnado un contexto (el desarrollo aeronáutico y el trabajo como escuderías), el diseño de los roles desarrollados por el alumnado es poco verosímil (no existen escuderías de globos aerostáticos) ni un motivo creíble para la necesidad de ese desarrollo de globos aerostáticos. Eso sitúa el proyecto en el nivel 2 de despliegue de la componente "Contexto". Incluir en la propuesta de contexto el propósito de preparar los globos aerostáticos para realizar experimentos meteorológicos habría sido un modo de incrementar a un nivel 3 para esa componente, que podría desarrollarse hasta un nivel 4 en caso de realizar realmente esos experimentos para un destinatario real con el que hubiera interacción. Aunque el Conflicto a resolver (conseguir que se eleve el globo) podría en el mundo real atender a muy distintos contenidos (diseño de materiales, aerodinámica, costes de los materiales) el diseño del proyecto orienta el trabajo sólo a lo relativo a los tipos, medida y cambios energéticos, de modo que hay una identificación entre el modo en que se propone al conflicto y los contenidos a desarrollar (los modelos y conceptos sobre energía). Por ese motivo, se ha situado el diseño del proyecto al nivel 4 de conflicto. Si el proyecto hubiera requerido, por ejemplo, la elaboración de un póster explicativo, esto habría implicado la participación de otros contenidos. En ese caso, aun siendo los contenidos nucleares en el proyecto, no serían los únicos necesarios, con lo que el conflicto se habría situado en el nivel 3.

En la propuesta de ese proyecto, el alumnado realiza los lanzamientos de globos aerostáticos, justificando a partir de modelos científicos el resultado obtenido (no se eleva porque se degrada mucha energía, la entrada de aire es demasiado ancha y entra aire frío que disminuye la energía cinética de las partículas,...) y argumenta qué decisiones técnicas deberían tomarse. Eso sitúa el proyecto en un nivel 3 de Discurso (debido al uso de pruebas) pero no llega a nivel 4, ya que ese nivel implicaría el desarrollo de dinámicas epistémicas (justificar cuán seguros estamos de nuestras conclusiones, desenvolverse en debates dirigidos al consenso sobre modelos, ...).

En cada etapa del proyecto Montgolfier tournament se construyen paulatinamente y formalizan los distintos conceptos sobre energía si bien algunos ellos se desarrollan sólo de forma parcial. Por ejemplo, en lo referente a los cambios energéticos, tal como se ha propuesto, el proyecto permite el desarrollo de las ideas de conservación, transformación y degradación de la energía, pero incide poco en la transmisión de energía. Por esa razón, se ubica el proyecto en el nivel 3 de despliegue de la componente Contenido. Si simplemente se hubiera pedido al alumnado que explicara cómo funciona un globo aerostático o construyera uno sin demasiada atención a formalizar los conceptos o aplicándolos acríticamente, nos hallaríamos respectivamente en los niveles 1 y 2 de despliegue del Contenido.

En este proyecto el alumnado no define ni el producto ni las etapas, que son cerrados, pero éstos permiten distintas soluciones tecnológicas, por lo que el proyecto se ha ubicado en el valor 2 de la componente de Apertura. Aunque no forman parte propiamente del Conflicto, y no se desarrollan en el proyecto, éste aplica distintos aspectos de las materias de Tecnología y Matemáticas. En los proyectos interdisciplinarios es habitual que una materia actúe de "tractora" y use contenidos desarrollados anteriormente en otras materias, como ocurre en este. Al estar enriquecido con otras dos materias además de las Ciencias, el proyecto se ha ubicado en el nivel 3 de interdisciplinariedad. 
Un análisis análogo ha permitido ubicar para los proyectos restantes el nivel para cada una de las componentes, y los resultados se muestran en la Figura 1. En ella se han representado los valores obtenidos para cada componente (tal como están especificadas en la Tabla 2) y se han unido los valores para cada componente de cada proyecto, resultando en una curva propia de cada proyecto.

Tabla 2. Rúbrica ABPMap

\begin{tabular}{|c|c|c|c|c|}
\hline & 1 & 2 & 3 & 4 \\
\hline Contexto & $\begin{array}{l}\text { El proyecto tiene } \\
\text { sólo sentido } \\
\text { dentro del aula. } \\
\text { No incorpora } \\
\text { formatos ni } \\
\text { elementos del } \\
\text { mundo real. } \\
\text { Contexto como } \\
\text { pretexto. }\end{array}$ & $\begin{array}{l}\text { Se incorporan } \\
\text { materiales o } \\
\text { voces del mundo } \\
\text { real (noticias, } \\
\text { formatos...). } \\
\text { Contexto y rol } \\
\text { del alumnado no } \\
\text { verosímil. }\end{array}$ & $\begin{array}{l}\text { El proyecto tiene } \\
\text { sentido en el } \\
\text { mundo real, del que } \\
\text { emerge e incorpora } \\
\text { elementos. El } \\
\text { contexto y los roles } \\
\text { del alumnado son } \\
\text { verosímiles. }\end{array}$ & $\begin{array}{l}\text { El proyecto } \\
\text { impacta en el } \\
\text { mundo real, } \\
\text { en el que } \\
\text { tiene sentido } \\
\text { y utilidad. El } \\
\text { contexto y } \\
\text { los roles del } \\
\text { alumnado son } \\
\text { reales. }\end{array}$ \\
\hline Conflicto & $\begin{array}{l}\text { El conflicto a } \\
\text { resolver no } \\
\text { instrumentaliza } \\
\text { los contenidos. } \\
\text { Podría resolverse } \\
\text { con contenidos } \\
\text { distintos sin } \\
\text { ningún problema. }\end{array}$ & $\begin{array}{l}\text { Los contenidos } \\
\text { están en la } \\
\text { periferia del } \\
\text { conflicto. Una } \\
\text { gran parte de } \\
\text { ellos se encuentra } \\
\text { fuera y se trata de } \\
\text { refilón. }\end{array}$ & $\begin{array}{l}\text { Los contenidos } \\
\text { están en el núcleo } \\
\text { del conflicto y son } \\
\text { esenciales para } \\
\text { resolverlo. Una } \\
\text { parte del conflicto } \\
\text { debe resolverse con } \\
\text { otros elementos. }\end{array}$ & $\begin{array}{l}\text { Contenidos y } \\
\text { conflicto están } \\
\text { identificados los } \\
\text { unos con el otro. }\end{array}$ \\
\hline Discurso & $\begin{array}{l}\text { La actividad de } \\
\text { los alumnos es de } \\
\text { juego búsqueda } \\
\text { y reproducción } \\
\text { de datos o } \\
\text { representación } \\
\text { de información, } \\
\text { sin ningún } \\
\text { proceso STEM } \\
\text { específico. }\end{array}$ & $\begin{array}{l}\text { Se aplican de } \\
\text { manera pautada } \\
\text { para la obtención } \\
\text { de datos procesos } \\
\text { y formatos propios } \\
\text { de las áreas } \\
\text { STEM (diseñar } \\
\text { experimentos, } \\
\text { hacer prototipos, } \\
\text { hacer conjeturas). }\end{array}$ & $\begin{array}{l}\text { Se aplican procesos } \\
\text { y formatos propios } \\
\text { de las áreas STEM } \\
\text { para argumentar } \\
\text { a partir de datos } \\
\text { y construir } \\
\text { conocimiento. }\end{array}$ & $\begin{array}{l}\text { Se aplican } \\
\text { dinámicas } \\
\text { epistémicas } \\
\text { propias de las } \\
\text { áreas STEM para } \\
\text { la evaluación y } \\
\text { validación de } \\
\text { conocimiento. }\end{array}$ \\
\hline $\begin{array}{l}\text { Contenidos } \\
\text { y modelos } \\
\text { científicos } \\
\text { STEM }\end{array}$ & $\begin{array}{l}\text { Los contenidos } \\
\text { son transmitidos } \\
\text { o reproducidos. }\end{array}$ & $\begin{array}{l}\text { Los contenidos } \\
\text { son aplicados y } \\
\text { desarrollados de } \\
\text { forma parcial e } \\
\text { informal. }\end{array}$ & $\begin{array}{l}\text { Los contenidos } \\
\text { son construidos } \\
\text { y desarrollados } \\
\text { de forma parcial } \\
\text { y formalizados } \\
\text { activamente. }\end{array}$ & $\begin{array}{l}\text { Los contenidos } \\
\text { son construidos } \\
\text { y desarrollados } \\
\text { de forma } \\
\text { completa y } \\
\text { formalizados. }\end{array}$ \\
\hline $\begin{array}{l}\text { Apertura y } \\
\text { autonomía } \\
\text { del } \\
\text { alumnado }\end{array}$ & $\begin{array}{l}\text { El proyecto } \\
\text { consiste en } \\
\text { una sucesión } \\
\text { de tareas } \\
\text { completamente } \\
\text { cerradas. }\end{array}$ & $\begin{array}{l}\text { Los alumnos } \\
\text { pueden tomar } \\
\text { alguna decisión } \\
\text { dentro de las } \\
\text { tareas y participan } \\
\text { en la evaluación a } \\
\text { partir de criterios } \\
\text { preestablecidos. }\end{array}$ & $\begin{array}{l}\text { Los alumnos } \\
\text { planifican la } \\
\text { consecución de } \\
\text { los objetivos, } \\
\text { deciden productos } \\
\text { y evaluación. }\end{array}$ & $\begin{array}{l}\text { Los alumnos } \\
\text { deciden temática } \\
\text { y planificación } \\
\text { del proyecto. }\end{array}$ \\
\hline $\begin{array}{l}\text { Inter-discipli- } \\
\text { nariedad }\end{array}$ & $\begin{array}{l}\text { Participa una sola } \\
\text { materia STEM. }\end{array}$ & $\begin{array}{l}\text { Participan dos } \\
\text { materias STEM. }\end{array}$ & $\begin{array}{l}\text { Participan tres } \\
\text { materias STEM. }\end{array}$ & $\begin{array}{l}\text { Participan cuatro } \\
\text { o más materias. }\end{array}$ \\
\hline
\end{tabular}


Las componentes didácticas o perfiles de proyectos no se asocian con el tipo de proyecto (Producto, Problema o Indagación). Se aprecian dos agrupaciones según el nivel de Contexto: tres proyectos con valores bajos de contexto y cinco proyectos con valores altos de contexto. Observamos que hay zonas de la rúbrica muy concurridas, en el nivel 2 de Apertura (por él discurren 7 de los 8 proyectos analizados) y el 3 de Contenidos. Estos podrían ser nodos que identifiquen espacios didácticos habituales (que podrían deberse no sólo a perspectivas didácticas del profesorado, sino también aspectos logísticos o relativos a la concepción del mundo escolar). La componente "Discurso" es la única que está representada por algún proyecto en todos sus niveles (si bien la mayoría sólo es representada por un proyecto (GeaTours y Mission to Stars). El resto de componentes son desplegadas sólo en algunos niveles de los 4 posibles. Sólo dos de los proyectos tienen valores bajos de Conflicto, Discurso y Contenidos, y son los que tienen mayor Apertura (Mission to Stars y GeaTours). El proyecto con valores más altos de Conflicto, Discurso y Contenidos (Karlsruhe) es el que menores valores presenta de Contexto, Apertura e Interdisciplinariedad. Esa oposición entre Contexto y Conflicto se repite en otro proyecto (Montgolfier tournament).

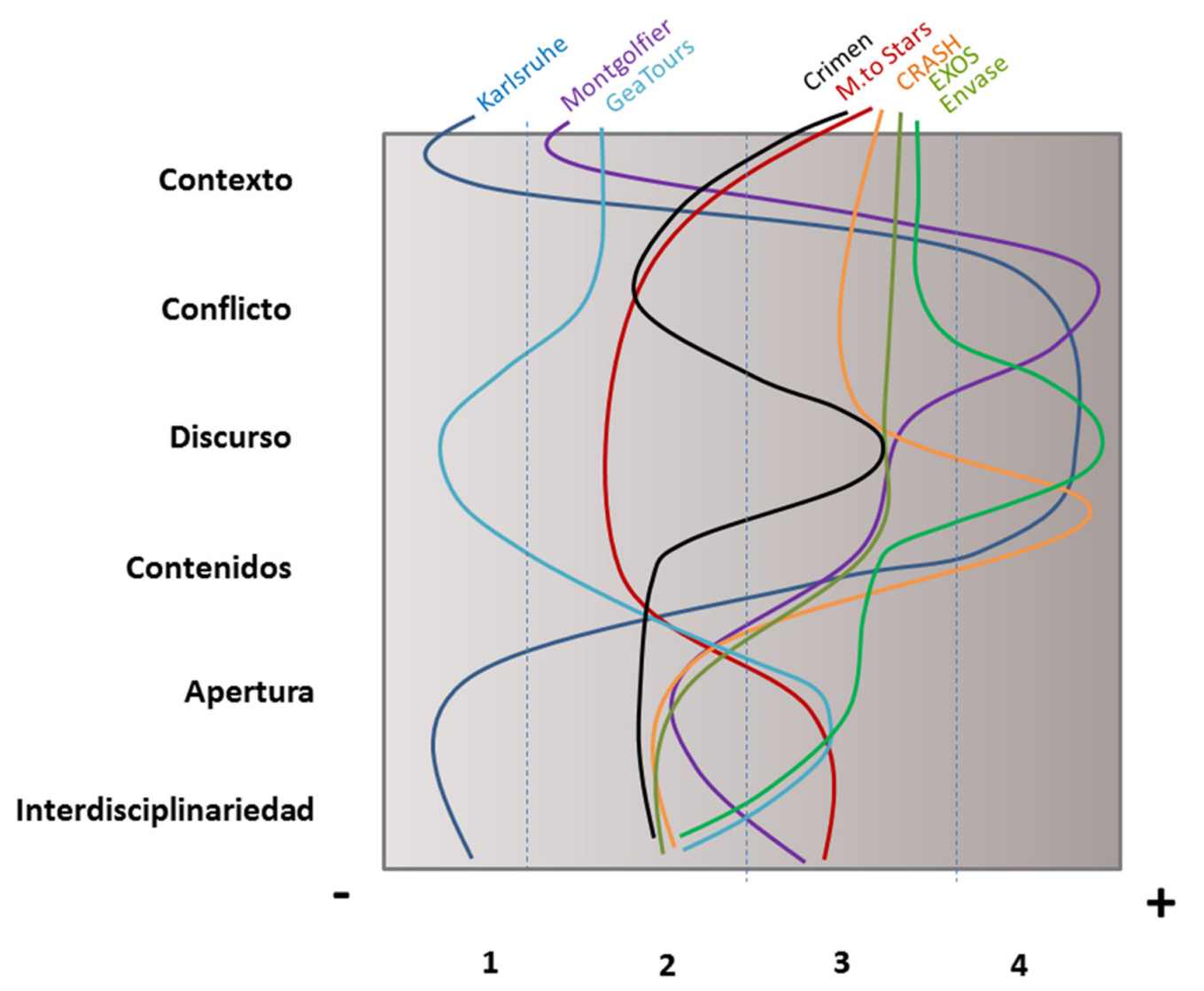

Figura 1.-Map ABPMap representando cada proyecto como una curva resultado de unir los valores obtenidos para cada componente de la rúbrica

\section{Conclusiones}

Estos primeros análisis con la rúbrica ABPMap indican que algo muy reivindicado en el $A B P$, la Apertura del proyecto, puede ir en contra del despliegue de Discurso, Conflicto y Contenidos. Igualmente, en contra de lo que se suele asumir, la Interdisciplinariedad no siempre va asociada a un mejor nivel de Contexto. Estas dos componentes didácticas (el 
Contexto y la Interdisciplinariedad), que suelen ser consideradas importantes en el desarrollo de ABP, no parecen ser buenas indicadoras del nivel de despliegue de otras componentes clave en el despliegue de la Competencia científica, como el Discurso, Conflicto y Contenido.

Parecen existir zonas de la rúbrica más concurridas que otras, lo que puede deberse a limitaciones metodológicas o logísticas de los espacios escolares (por ejemplo, las dificultades para el trabajo interdisciplinar en centros donde la formación se ofrece repartida en materias, o las complejidades relativas a la custodia y organización de excursiones para hacer que los alumnos y proyectos salgan a contextos fuera del mundo escolar). De todas ellas, la más concurrida es la del nivel 2 de Apertura, una componente muy relacionada con aspectos -como la evaluación o autonomía del alumnado- muy sensibles a visiones pedagógicas no específicas de las STEM.

Los resultados obtenidos indican que ABPMap puede ser una herramienta útil para el análisis de un número amplio de proyectos para identificar zonas concurridas y perfiles didácticos habituales de proyectos. Esto puede ser de utilidad para, en segunda instancia, asociar cada perfil a sus resultados de aprendizaje e identificar "formas didácticas" de éxito para el despliegue de la Competencia científica o la necesidad de "despejar" dificultades logísticas u organizativas que puedan existir para el despliegue de componentes que identificamos como importantes para la competencia científica. Así mismo, puede constituir una herramienta de evaluación y mejora del diseño de actividades de Aprendizaje Basado en Proyectos o para la transformación de actividades previas en proyectos. En todo caso estos resultados y conclusiones son únicamente prospectivos sobre ocho proyectos, y sería necesario ampliar el análisis incluyendo más proyectos de distintos tipos, distintos autores y distintos niveles de experiencia en el diseño de propuestas.

\section{Agradecimientos}

Reflexiones incluidas en este artículo se enmarcan en la reflexión metodológica del grupo de investigación consolidado ACELEC (Activitat Científica Escolar: Llenguatges, Eines i Contextos) referencia 2017 SGR 1399) por AGAUR y financiado por el Ministerio de Economía y Competitividad (referencia EDU2015-66643-C2-1-P).

\section{Referencias bibliográficas}

Acevedo-Díaz, J.A. (2004). Reflexiones sobre las finalidades de la enseñanza de las ciencias: educación científica para la ciudadanía. Revista Eureka sobre Enseñanza y Divulgación de las Ciencias, 1(1), 3-16.

Acevedo-Díaz, J. A. y García-Carmona, A. (2017). Controversias en la historia de la ciencia y cultura científica. Madrid: Los Libros de la Catarata.

Albalat, A. (2017). Design thinking en STEAM. Revista Ciències, 34, 29-34.

Blanco A., España E. y Rodríguez F. (2012). Contexto y enseñanza de la competencia científica. Alambique, Didáctica de las Ciencias Experimentales, 70, 9-18.

Bravo, B., Puig, B. y Jiménez-Aleixandre, P. (2009). Competencias en el uso de pruebas en argumentación. Educación Química, 20(2), 137-142.

Brown, R., Brown, J., Reardon, K. y Merrill, C. (2011). Understanding STEM: Current perceptions. Technology and Engineering Teacher 70(6), 5-9.

Bybee, R.W. (1997). Achieving scientific literacy: From purposes to practices. Portsmouth: Heinemann. 
Bybee, R. W. (2010). What is STEM Education? Science, 329 (5995), 996-996.

Caamaño, A. (2011). Contextualización, indagación y modelización. Tres enfoques para el aprendizaje de la competencia científica en las clases de química. Aula de Innovación Educativa, 207, 17-21.

Couso, D. (2014). De la moda de "aprender indagando" a la indagación para modelizar: una reflexión crítica. XXVI Encuentro de Didáctica de las Ciencias Experimentales. Huelva (Andalucía).

Couso, D. (2015). La clau de tot plegat: la importancia de "què" ensenyar a l'aula de ciències. Revista Ciències, 29, 29-36.

Couso, D. (2017). Perquè estem a STEM? Definint I'alfabetització STEM per a tothom i amb valors. Revista Ciències, 34, 21-29.

Díaz N. y Jiménez-Liso M. R. (2012). Las controversias sociocientíficas: temáticas e importancia para la educación científica. Revista Eureka sobre Enseñanza y Divulgación de las Ciencias, 9(1), 54-70.

Domènech-Casal, J. (2014). GeaTours: una experiència geolòTICa d'aprenentatge cooperatiu que promou la indagació i l'autoregulació dels aprenentatges. Revista Ciències 27, 2-8.

Domènech-Casal, J. (2017a). Aprendizaje Basado en Proyectos y competencia científica. Experiencias y propuestas para el método de estudios de caso. $X$ Congreso Internacional sobre Investigación en Didáctica de las Ciencias, Sevilla 2017.

Domènech-Casal, J. (2017b). Aprenentatge Basat en Projectes en àmbits STEM. Claus metodològiques i reptes. Revista Ciències, 33, 2-7.

Domènech-Casal, J (2017c). Proyectando BioGeo, un itinerario de trabajo por proyectos contextualizados basado en la indagación y la Naturaleza de la Ciencia. Alambique, Didáctica de las Ciencias Experimentales, 89, 54-61.

Domènech-Casal, J. (2018a). Comprender, Decidir y Actuar: una propuesta-marco de Competencia Científica para la Ciudadanía. Revista Eureka sobre Enseñanza y Divulgación de las Ciencias, 15 (1), 1105.

Domènech-Casal, J. (2018b). Concepciones de alumnado de secundaria sobre energía. Una experiencia de Aprendizaje Basado en Proyectos con globos aerostáticos. Enseñanza de las Ciencias, 36(2), 191-213.

Domènech-Casal, J. (2019). Retorno a Karlsruhe: una experiencia de indagación con la Tabla Periódica para aprender la estructura y propiedades de los elementos. Revista Eureka de Enseñanza y Divulgación de las Ciencias, 16(1), 1201, 1-18.

Domènech-Casal, J., Couso, D., Pérez, M., Márquez, C. (2018a). Propósito, contexto e conteúdo no ensino de ciências. Revista Pátio Ensino Medio, Profissional e Tecnológico, 38. 10-13.

Domènech-Casal, J., Gasco, J., Royo, P. y Vilches, S. (2018b). CRASH: un proyecto de enseñanza de cinemática y dinámica en el contexto del análisis pericial de accidentes. Revista Eureka sobre Enseñanza y Divulgación de las Ciencias, 15 (2), 2103, 1-17.

Domènech-Casal, J. y Ruiz-España, N. (2017). Mission to Stars: un Proyecto de Investigación alrededor de la astronomía, las misiones espaciales y la investigación científica. Revista Eureka de Enseñanza y Divulgación de las Ciencias, 14 (1), 98-114. 
Duranti, A. y Goodwin, C. (Eds). (1992). Rethinking context: Language as an interactive phenomenon. Cambridge: Cambridge, University Press

García-Molina, R. (2015). Pseudociencia en el mundo contemporáneo. Alambique, Didáctica de las Ciencias Experimentales, 81, 25-33.

Gilbert, J.K. (2006). On the nature of "context" in chemical education. International Journal of Science Education, 28(9), 957-976.

Gilbert, J. K., Bulte, A.M.W. y Pilot, A. (2011). Concept development and transfer in context-based science education. International Journal of Science Education, 33(6), 817-837.

Grau, R. (2009). Altres formes de fer ciència. Alternatives a l'aula de secundària. Rosa Sensat, Barcelona.

Kilpatrick, W.E. (1918). The Project Method: the use of the purposeful act in the educative process. New York: Teachers college, Columbia University.

Kolst $\varnothing$, S.D. (2001). Scientific Literacy for Citizenship: Tools for Dealing with the Science Dimension of Controversial Socioscientific Issues. Science Education, 85(3), 291-310.

Kortland, J. (2007). Context-based science curricula: Exploring the didactical frictions between context and science content. ESERA. Malmö.

Larmer, J., Mergendoller, J. y Boss, S. (2015). Setting the Standard for Project Based Learning: A Proven Approach to Rigorous Classroom Instruction. ASCD, Alexandria.

López, V., Domènech-Casal, J. (2018). Juegos y gamificación en las clases de ciencia: ¿una oportunidad para hacer mejor clase o para hacer mejor ciencia? Revista Eletrônica Ludus Scientiae (RELUS), 2(1), 35-44.

Martín, X. (2016). Proyectos con alma. Graó: Barcelona.

Miró, M., Saperas, A.M., Tarragó, M., Tordera, M.R. y Domènech-Casal, J. (2016). Cinc experiències i reflexions metodològiques sobre l'Aprenentatge Basat en Projectes a les Ciències. Revista Ciències, 32, 23-33.

OCDE (2013). PISA 2015 draft science framework. http://www.oecd.org/

Planella, M., Vilches, S., Font, J., Grau, R. y Gorriz, M. (2017) Espai, context i creativitat. Aprenentatge de les matemàtiques. Revista Ciències, 33, 42-48.

Redondo, M., Ruiz, N. Sánchez, S., Solé, R., Calvet, J. y Caño, M. (2017). Context, entorn i servei: l'aprenentatge mitjançant projectes, de les àrees científiques a la interdisciplinarietat. Revista Ciències, 33, 49-56.

Rocard, M., Csermely, P., Jorde, D. Lenzen, D., Walwerg-Heriksson, H. y Hemmo, V. (2006). Science Education Now: a new pedagogy for the future of Europe. Report for the European Comission.

Ruiz, N., Llorente, I. y Domènech-Casal, J. (2017). Indagación, Exoplanetas y Competencia Científica. Los Estudios de Caso como ABP para las Ciencias. Enseñanza de las Ciencias de la Tierra, 25(2), 191-202.

Sadler, T. D. (2009). Situated learning in science education: socio-scientific issues as contexts for practice. Studies in Science Education, 45(1), 1-42.

Sanmartí, N. (2016). Trabajo por proyectos: ¿Filosofía o metodología? Cuadernos de Pedagogía, 472, 44-46. 
Sanmartí, N., Burgoa, B. y Nuño, T. (2011) ¿Por qué el alumnado tiene dificultades para utilizar sus conocimientos escolares en situaciones cotidianas? Alambique, Didáctica de las Ciencias Experimentales, 67, 62-68.

Sanmartí, N. y Márquez, C. (2017). Aprendizaje de las ciencias basado en proyectos: del contexto a la acción. Ápice, Revista de Educación Científica, 1(1), 3-16.

Simarro, C. y Couso, D. (2016). Análisis de una actividad tinkering en el marco de la educación STEM. Comunicación y Pedagogía, 291-292, 65-7.

Vieira, M.R., Tenreiro-Vieira, C. y Martins, E. (2010). Pensamiento crítico y literacia científica. Alambique, Didáctica de las Ciencias Experimentales, 65, 96-104.

Vilches A., Gil D. y Cañal P. (2010). Educación para la sostenibilidad y educación ambiental. Investigación en la Escuela, 71, 5-15.

Windschitl, M., Thompson, J. y Braaten, M. (2008). Beyond the scientific method: Modelbased inquiry as a new paradigm of preference for school science investigations. Science Education, 92, 941-962 